\title{
Investigation of photodamage by femtosecond laser to cells via gold nanorods
}

\author{
Yang Li, Hao He*, Huanyu Song, Bowen Liu, \\ Minglie $\mathrm{Hu}$ and Chingyue Wang \\ Ultrafast Laser Laboratory \\ Key Laboratory of Optoelectronic Information Technology \\ (Ministry of Education) \\ College of Precision Instrument and Optoelectronics Engineering \\ Tianjin University, Tianjin 300072, P. R. China \\ *haohe@sjtu.edu.cn
}

Received 11 December 2015

Accepted 29 February 2016

Published 11 April 2016

\begin{abstract}
Usually, only focused femtosecond (fs) lasers at near-infrared (NIR) range can induce photodamage to transparent cells, making it difficult to treat large amount of cells by such optical methods for photostimulation. In this study, we clarify the mechanism of photodamage to cells that are co-cultured with gold nanorods (GNRs) by fs laser. The pulse duration and repetition rate of the fs laser play a key role in cell damage suggesting that the heat accumulation contributes to the major part for the cell damage rather than the high peak power which mainly determines the efficiency of multiphoton excitation. We further show that cellular $\mathrm{Ca}^{2+}$ can also be released in this scheme, but the process is more sensitive to peak power. Our results can provide a large-scale GNR-mediated photostimulation for cell signaling modulation.
\end{abstract}

Keywords: Nanorods; photothermal effect; thermo-optical materials; ultrafast lasers.

\section{Introduction}

Nanoparticles have been demonstrated and experimentally realized for a long time with great advantages for biological and medical applications. In the past decades, significant progress has been made with nanoparticles on tissue imaging, sensing, drug delivery and therapy. ${ }^{1,2}$ The functions and applications of nanoparticles can be further improved and extended when combined with lasers by their strong response to photons. For example, nanoparticles can work for delivering and releasing drugs efficiently or directly providing damage effect to targeted cells or tissue when excited by lasers. ${ }^{3-5}$ Gold nanorods (GNRs) are an important type of

*Corresponding author.

This is an Open Access article published by World Scientific Publishing Company. It is distributed under the terms of the Creative Commons Attribution 4.0 (CC-BY) License. Further distribution of this work is permitted, provided the original work is properly cited. 
nanoparticles since their toxicity to cells is relatively low due to their chemical stability. ${ }^{6}$ They can be easily synthesized with designed size and shape which determines their spectral response and extinction efficiency. ${ }^{7}$

As the classic "optical window", photons in the near-infrared (NIR) band can hardly stimulate cells due to the relatively low absorption. GNRs with specially designed shape can stain cells with minimal damage and enhance response to photons at NIR range. ${ }^{7-9}$ Therefore, GNRs are widely used as photosensitizers due to their high absorption to photons to generate heating effect. The heat will physically disturb cell processes, damage molecules and cellular structures, and may stimulate mitochondria to release ROS. ${ }^{10,11}$ In this regard, apoptosis or necrosis can be induced by such high ROS level. ${ }^{12}$ If the heating is too intense, cells will be killed directly.

Several theories on the mechanism of laser exciting nanoparticles have been investigated. It is not too difficult to heat nanoparticles as long as the laser wavelength matches the extinction spectrum of nanoparticles. ${ }^{13}$ When applied to cells, an important issue is to strictly confine the thermal damage to targeted cells while minimizing the side effect to surroundings. Therefore, ultrashort pulsed lasers are used to minimize heating diffusion by decreasing linear accumulation of photothermal heating while still inducing effective heating and transient high temperature to GNRs in targeted cells. According to previous reports, those ultrashort laser pulses heat nanoparticles very fast and then release thermal energy in the form of tiny shockwave, instead of releasing heat linearly to a large area in the case of continuouswave laser irradiation. ${ }^{14}$ The physical mechanism of GNRs responding to laser irradiation is complicated, and it is believed that some nonlinear and plasmonic processes are simultaneously involved. ${ }^{15-17}$ The two-photon luminescence (TPL) from GNRs excited by femtosecond (fs) lasers suggests that the multiphoton process may also take place. ${ }^{18-20}$ Here, the frequency of incident photons matches the eigenfrequency of electron oscillation in the GNR's surface that can induce surface plasmon resonance (SPR). Hence, the ultrahigh peak power of fs laser pulses can easily excite intense electron oscillation in the surface of GNRs, which may then absorb more energy from incident photons and release energy via photon radiation or thermal relaxation. In this regard, the nonlinear processes in GNRs could also contribute to cell damage when irradiated by fs lasers. To clarify the processes of photons exciting GNRs, we utilized fs lasers with different pulse durations and repetition rates to study cell damage with GNRs at different conditions. All cells in an area with a 1-mm diameter were irradiated simultaneously. By investigating the key factor that dominates GNRmediated cell damage with tunable fs lasers, we found that heat accumulation, determined by pulse duration and repetition rate, plays a key role in cell damage rather than the peak power of laser pulses. The GNR-enhanced cellular sensitivity to photons could be then applied to large-scale cell signaling modulation.

\section{Experimental Methods}

To scale cell viability, mitochondrial membrane potential (MMP), an important and classic indicator of initial apoptosis and fast necrosis, is probed here to evaluate cell damage by labeling mitochondrial membrane with JC-1 and TMRM (life technology). ${ }^{21,22}$ Generally, the significant and irreversible decrease of MMP indicates death of cells (slow and fast decrease correspond to apoptosis and necrosis, respectively). ${ }^{23,24}$ To be simple, in this study, cell damage was indicated by MMP level without differentiating apoptosis or necrosis.

HeLa cells were cultivated in Dulbecco's modified Eagle's medium containing $10 \%$ fetal bovine serum, $2 \mathrm{mM} \mathrm{L-glutamine}$, and $1 \%(\mathrm{v} / \mathrm{v})$ penicillin/streptomycin at $37^{\circ} \mathrm{C}$ with $5 \% \mathrm{CO}_{2}$. Before microscopic experiments, cells were seeded in $35 \mathrm{~mm}$ glassbottom $(0.17 \mathrm{~mm}$ thick $)$ dishes, and cultured with GNRs (Nanopartz Inc.) at $120 \mathrm{pM}$ for $36 \mathrm{~h}$ to minimize the transient damage effect of GNRs. ${ }^{25}$ The length and width of GNRs here were $65 \mathrm{~nm}$ and $10 \mathrm{~nm}$, respectively, with a length/width ratio 6.5 corresponding to the SPR peak at $1045 \mathrm{~nm}$. The surface of GNRs was coated with amino acid to minimize the toxicity to cells. When staining cells, GNRs could diffuse through cell membrane with moderate damage to cells by affecting permeability of cellular membrane or endocytosis process. ${ }^{26,27}$ Hence, the concentration of GNRs could be neither too low (to maintain good sensitivity of cells to laser irradiation) nor too high (to protect cells from toxicity of GNRs). 


\section{Results and Discussions}

Co-culturing with GNRs is relatively safe to cells. The viability of cells after 36 -h staining with GNRs was measured as presented in Fig. 1(a). It can be found that those cells were quite healthy compared with control group.

Without GNRs, laser irradiation at NIR band to cells could hardly provide any stimulation to them due to the relatively low absorption. In contrast, cells would suffer photothermal damage when irradiated by fs lasers with GNRs inside, which was mainly determined by the parameters of the laser. The damage effect to cells could not be simply evaluated by the total laser energy (irradiation dose). For example, when cells were exposed to lasers with the same total energy, the high-power and short-time irradiation may cause much more serious cell damage than the low-power but longtime treatment. The situation became much more complicated when more parameters of fs lasers were involved. The pulse duration and repetition rate determined the heating and thermal accumulation/ release process, while the peak power in a pulse influenced the mechanism of heat generation and nonlinear excitation efficiency. It should be noted that such parameters may not be totally independent from each other. Here, we utilized fs-pulse $\mathrm{Yb}^{3+}$-doped fiber amplifier at $1040 \mathrm{~nm}$ for cell damage study with GNRs to clarify the actual process. The laser beam was focused to a spot with diameter at $1 \mathrm{~mm}$ for cell irradiation. To test

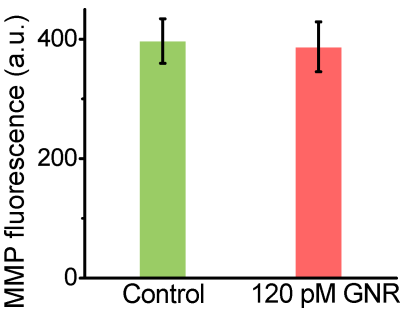

(a)

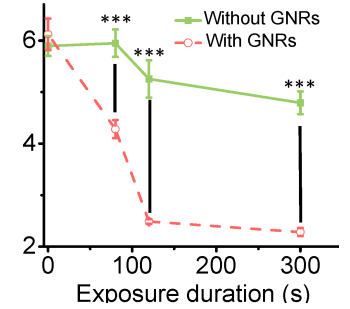

(b)
Fig. 1. Cell viability assay with GNRs: (a) Hela cells stained with GNRs at $120 \mathrm{pM}$ for $36 \mathrm{~h}(n=29)$ showed no toxicity response compared with the control group $(n=38)$. No statistical difference. (b) Cells with GNRs were very sensitive to laser irradiation $(0.55 \mathrm{~W}, 1 \mathrm{MHz}, 134 \mathrm{fs})$ with different irradiation durations ( $n=46,45,46$, and 71 , respectively, for four different irradiation durations $(0,80,120$, and $300 \mathrm{~s}))$. In control group, there was only a moderate decrease of MMP ( $n=40,64,27$, and 42, respectively, for four irradiation conditions). Two groups at each exposure duration, ${ }^{* * *} P<0.0001$, by Student's test. effectiveness of such GNRs-mediated photodamage, the amplifier was tuned to provide an output at 134fs pulse with $0.55-\mathrm{W}$ average power at $1-\mathrm{MHz}$ repetition rate. As shown in Fig. 1(b), MMP decrease was proportional to irradiation duration, indicating such laser irradiation intensity could significantly damage cells. As a control, cells without GNRs showed no significant response to such laser treatments.

To investigate the contribution of peak power of laser pulses to cell damage by laser irradiation, the pulse duration of the laser was tuned to $60 \mathrm{fs}$ and $600 \mathrm{fs}$, respectively. Cells with GNRs, divided to two groups, 60-fs and 600-fs group, were then treated at the same mean power of $0.5 \mathrm{~W}(55 \mathrm{~W} /$ $\left.\mathrm{cm}^{2}\right)$ both for $90 \mathrm{~s}\left(6 \times 10^{3} \mathrm{~J} / \mathrm{cm}^{2}\right)$. In this way, the two cell groups consumed the same pulse energy, mean power, and total energy. The differences of the laser parameters in those two groups were hence only pulse duration and peak power, inducing different peak powers and thermal effects. Here, we considered that the nonlinear processes that could contribute to cell damage, if any, was determined by peak power of laser pulses because they mostly rely on transient photon density. ${ }^{15,28}$ As shown in Fig. 2, cells in 600-fs group irradiated with 10-fold lower peak power of laser pulses showed more MMP decrease, indicating much higher cell damage. The thermal accumulation of $600-$ fs pulses was stronger than that of 60-fs pulses due to higher duty cycle, ${ }^{29,30}$ but the nonlinear effect by the $600-\mathrm{fs}$ pulses was much weaker than that by 60 -fs pulses. Therefore, this result suggests that heat accumulation may be the dominant contribution to cell damage rather than nonlinear effects when heated with relatively low-power fs laser.

Repetition rate of laser pulse train is another important factor for heat accumulation. To clarify the influence of this parameter, another fs-pulse fiber amplifier also at $1040 \mathrm{~nm}$ but with a $36-\mathrm{MHz}$ repetition rate (pulse duration: $134 \mathrm{fs}$ ) was used here to further investigate cell response to different laser irradiations with GNRs. At first, a group of cells (Group 1) was irradiated by the $1-\mathrm{MHz}$ laser (the pulse duration was tuned to 134 fs to be consistent with the $36-\mathrm{MHz}$ laser) at $0.13 \mathrm{~W}$ for $800 \mathrm{~s}$ while the second cell group (Group 2) was treated with the $36-\mathrm{MHz}$ laser at $4.64 \mathrm{~W}$ for $22.23 \mathrm{~s}$, which ensured cells in those two groups suffering the same laser pulses (the same pulse energy, pulse duration, and peak power), pulse number, and total energy. 


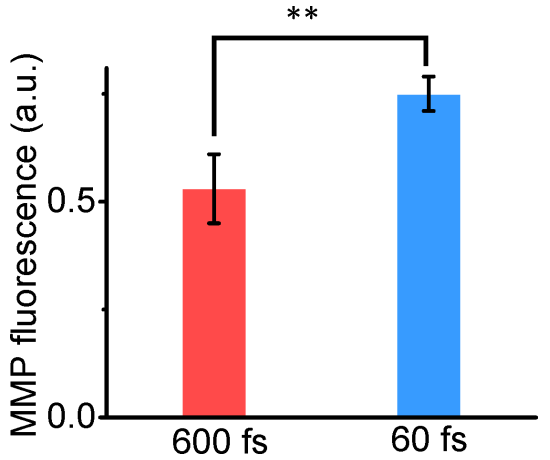

(a)

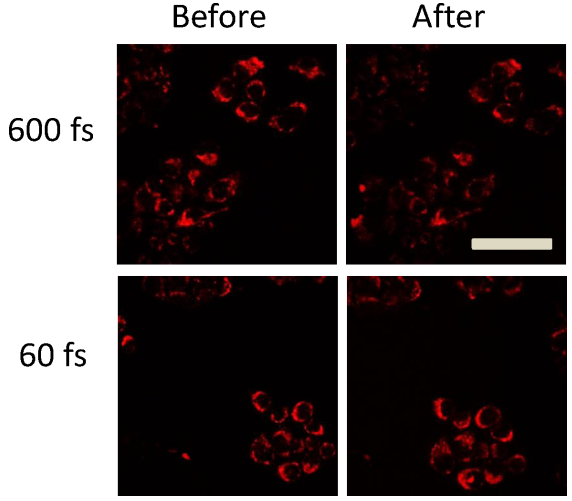

(b)

Fig. 2. Cell damage depends on pulse duration. Cells were treated with an fs laser at $0.5 \mathrm{~W}$ with $1-\mathrm{MHz}$ repetition rate for $90 \mathrm{~s}$ with different pulse durations at $600 \mathrm{fs}(n=17)$ and $60 \mathrm{fs}(n=31)$, respectively. The cellular MMP in $600-\mathrm{fs}$ group showed higher decrease. ${ }^{* *} P<0.001$, by Student's test. Down: fluorescence photos of cellular MMP. Bar: $50 \mu \mathrm{m}$.

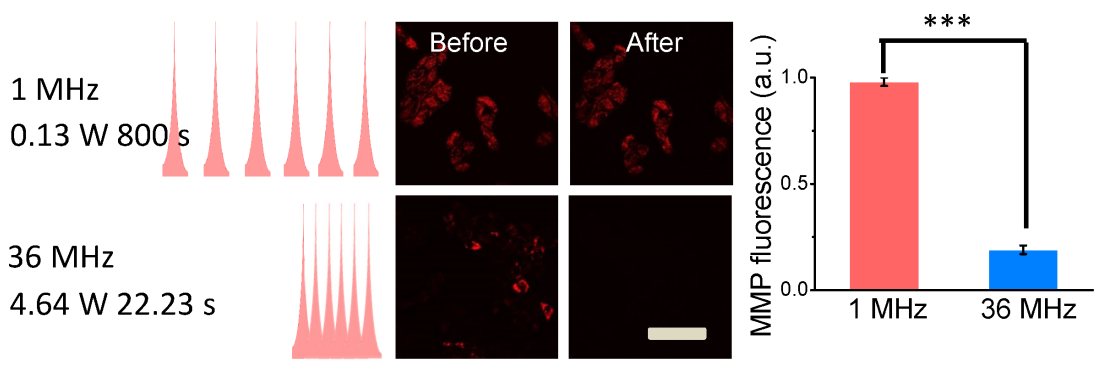

(a)

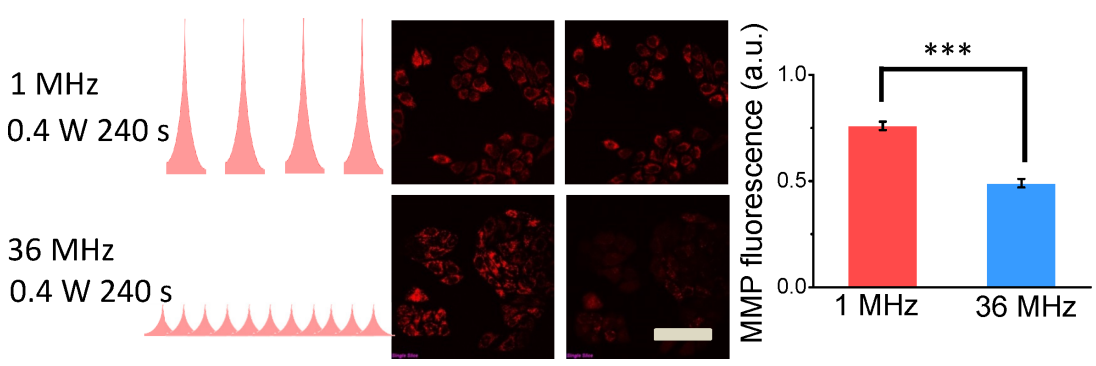

(b)
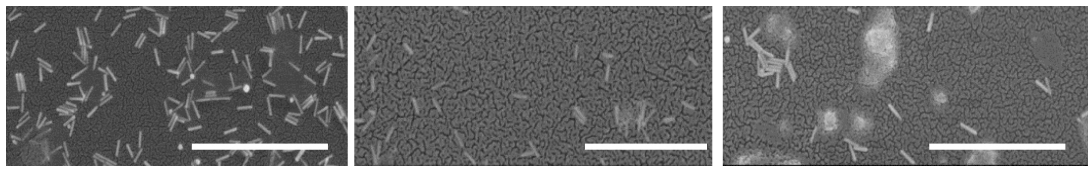

(c)

Fig. 3. Cell damage was quite dependent on repetition rate. More MMP decrease could be found with laser irradiation with higher repetition rate, with no matter the same pulses $\left((\mathrm{a}),{ }^{* * *} P<0.0001\right.$, by Student's test.) or the same mean power and total energy from 1-MHz and 36-MHz lasers ((b), ${ }^{* * *} P<0.0001$, by Student's test.). Bar: $50 \mu \mathrm{m}$. (c) Scanning electron microscopy images of GNRs. Left: without laser treatment; Middle: GNRs in Group 2; Right: GNRs in Group 4. No melt or shape changes of GNRs were observed. Bar: $1 \mu \mathrm{m}$. 
Table 1. Cells were treated with fs lasers with different parameters.

\begin{tabular}{cccccc}
\hline Cell group & Repetition rate & Power & Irradiation duration & Pulse duration & Total energy \\
\hline 1 & $1 \mathrm{MHz}$ & $0.13 \mathrm{~W}$ & $800 \mathrm{~s}$ & $134 \mathrm{fs}$ & $103 \mathrm{~J}$ \\
2 & $36 \mathrm{MHz}$ & $4.64 \mathrm{~W}$ & $22.23 \mathrm{~s}$ & $134 \mathrm{fs}$ & $103 \mathrm{~J}$ \\
3 & $1 \mathrm{MHz}$ & $0.4 \mathrm{~W}$ & $240 \mathrm{~s}$ & $134 \mathrm{fs}$ & $96 \mathrm{~J}$ \\
4 & $36 \mathrm{MHz}$ & $0.4 \mathrm{~W}$ & $240 \mathrm{~s}$ & $134 \mathrm{fs}$ & $96 \mathrm{~J}$ \\
\hline
\end{tabular}

In this way, the only difference of laser irradiation between Groups 1 and 2 was the repetition rate. As shown in Fig. 3(a), more significant cell damage in Group 2 could be found while Group 1 suffered very little damage, indicating heat accumulation played a key role in such photodamage process. It could be observed in Group 2 that cell membrane was greatly damaged directly according to the white-light microscopy. For further verification, one more cell group (Group 3) was irradiated by the $1-\mathrm{MHz}$ laser at $0.4 \mathrm{~W}$ for $240 \mathrm{~s}$ and the last group (Group 4) of cells was irradiated by the $36-\mathrm{MHz}$ laser also at $0.4 \mathrm{~W}$ for $240 \mathrm{~s}$. In this case, the mean power and total energy of two lasers were kept the same but pulses and repetition rate were different. Interestingly, cells in Group 4 again showed greater damage (Fig. 3(b)), even though Group 3 suffered 36-fold higher peak power and larger pulse energy. Parameters of laser irradiation for these four groups are listed in Table 1. Therefore, it can be concluded that repetition rate and pulse duration, which determine the heat accumulation effect, dominate cell damage under fs-laser irradiation with GNRs. It should be noted here that in all experiments, in this study, GNRs were not melt during laser treatment (Fig. 3(c)) since the laser power/energy used here was still relatively low, which is also in good agreement with previous studies. ${ }^{31-33}$

Cellular response to such laser stimulation is actually more complicated than simple cell damage. Cellular molecules could be also influenced by such stimulation. We show here that such stimulation can be used for excitation of cellular $\mathrm{Ca}^{2+}$ increase but with a different mechanism from cell damage. With the $36-\mathrm{MHz}$ laser at first, cells were treated at $2.32 \mathrm{~W}$ for $6 \mathrm{~s}$ but no $\mathrm{Ca}^{2+}$ increase was observed. However, if the power was boosted to $4.64 \mathrm{~W}$, significant $\mathrm{Ca}^{2+}$ increase could be found in all irradiated cells for only $3 \mathrm{~s}$ (remaining the same total energy) as shown in Fig. 4(a). Even if the exposure duration was decreased to $1 \mathrm{~s}$, significant $\mathrm{Ca}^{2+}$ increase still could be found. Therefore, we believe that $\mathrm{Ca}^{2+}$ increase is also sensitive to peak power of laser pulse. To confirm this point, cells were then

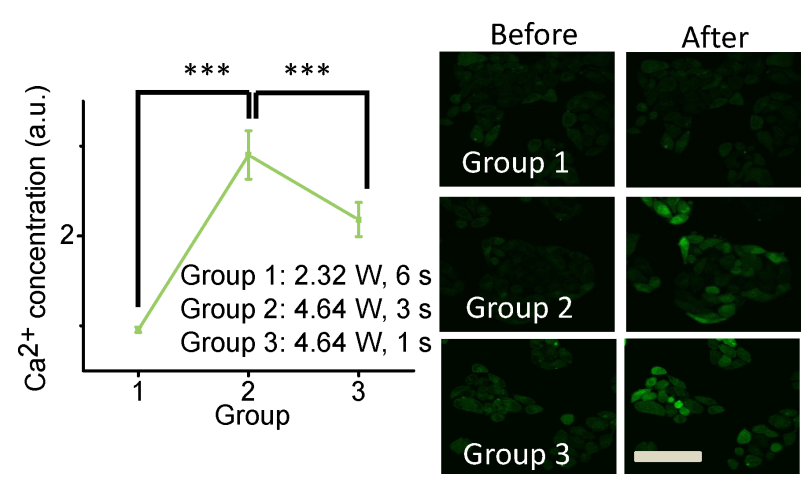

(a)

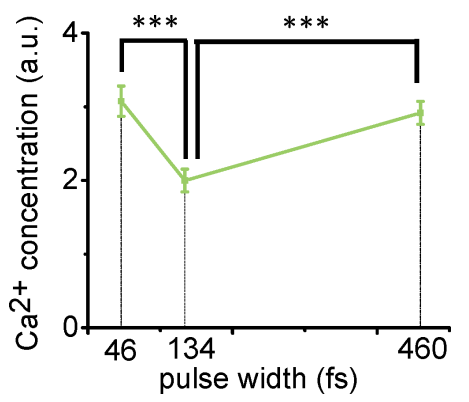

(b)

Fig. 4. Laser irradiation could induce global $\mathrm{Ca}^{2+}$-level increase in GNR-stained cells. (a) Cells were treated with the 36-MHz lasers at different conditions $\left(n=66,48\right.$ and 56 , respectively in such three different groups). There were nearly no $\mathrm{Ca}^{2+}$-increase in Group 1 but significant $\mathrm{Ca}^{2+}$-increase in Groups 2 and 3, indicating $\mathrm{Ca}^{2+}$-increase was partially-dependent on peak power. ${ }^{* * *} P<0.0001$, by Student's test. (b) Cells were treated with the $1-\mathrm{MHz}$ laser at $0.38 \mathrm{~W}$ for $240 \mathrm{~s}$ with different pulse duration. ( $n=31$ at $46 \mathrm{fs}, n=32$ at $134 \mathrm{fs}$, and $n=31$ at $460 \mathrm{fs}$, respectively). ${ }^{* * *} P<0.0001$, by Student's test. Ca ${ }^{2+}$ increased less if the pulse duration increased suggesting the dependence on peak power, and then again highly increased if the pulse duration was long enough, indicating the dependence on thermal accumulation. Bar: $50 \mu \mathrm{m}$. 
treated with the $1-\mathrm{MHz}$ laser at $0.38 \mathrm{~W}$ for $240 \mathrm{~s}$ with 134-fs and 46-fs pulse duration, respectively, which has the same pulse energy but about threefold difference in peak power. It can be found in Fig. 4(b), the 46-fs laser induced much higher $\mathrm{Ca}^{2+}$ level increase than the 134-fs laser, suggesting that $\mathrm{Ca}^{2+}$ increase is more dependent on peak power. If the pulse duration was tuned to $460 \mathrm{fs}, \mathrm{Ca}^{2+}$ concentration then returned back to higher level because the thermal effect was too large. Therefore, cellular $\mathrm{Ca}^{2+}$ increase is partially-dependent on peak power when the thermal effect is not too high.

\section{Conclusions}

In conclusion, we show that repetition rate and pulse duration of fs-laser pulses that determine heat accumulation in laser irradiation to GNRs play a key role in cell damage. Lasers with longer pulse duration and higher repetition rate induce more MMP depolarization at the same mean power and total energy. Such stimulation can be used for cellular $\mathrm{Ca}^{2+}$ increase which is found partially-dependent on peak power of laser pulses. Our results thus provide a better understanding for excitation to GNRs by fs lasers and an optical method for largescale cellular $\mathrm{Ca}^{2+}$-modulation, which can be applied for activation of tissue $\mathrm{Ca}^{2+}$ signaling and thus facilitate optical engineering of gene expression.

\section{Acknowledgments}

This work was supported by grants from National Natural Science Foundation of China (NSFC) 81571719, 61322502, Open Project of State Key Laboratory of Modern Optical Instrumentation, Zhejiang University, and Program for Changjiang Scholars and Innovative Research Team in University IRT13033.

\section{References}

1. L. Tong, J.-X. Cheng, "Gold nanorod-mediated photothermolysis induces apoptosis of macrophages via damage of mitochondria," Nanomedicine 4, 265-276 (2009).

2. S. Akhter, M. Z. Ahmad, F. J. Ahmad, G. Storm, R. J. Kok, "Gold nanoparticles in theranostic oncology: Current state-of-the-art," Expert Opin. Drug Deliv. 9, 1225 (2012).
3. J. A. Webb, R. Bardhan, "Emerging advances in nanomedicine with engineered gold nanostructures," Nanoscale 6, 2502 (2014).

4. M. Perez-Hernandez, P. Del Pino, S. G. Mitchell, M. Moros, G. Stepien, B. Pelaz, W. J. Parak, E. M. Galvez, J. Pardo, J. M. de la Fuente, "Dissecting the molecular mechanism of apoptosis during photothermal therapy using gold nanoprisms," ACS Nano 9, $52(2015)$.

5. J. R. Melamed, R. S. Edelstein, E. S. Day, "Elucidating the fundamental mechanisms of cell death triggered by photothermal therapy," $A C S$ Nano 9, 6 (2015).

6. N. Khlebtsov, L. Dykman, "Biodistribution and toxicity of engineered gold nanoparticles: A review of in vitro and in vivo studies," Chem. Soc. Rev. 40 (3), 1647-1671 (2011).

7. J. Pérez-Juste, I. Pastoriza-Santos, L. M. LizMarzán, P. Mulvaney, "Gold nanorods: Synthesis, characterization and applications," Coord. Chem. Rev. 249, 1870-1901 (2005).

8. X. Huang, P. K. Jain, I. H. El-Sayed, M. A. ElSayed, "Plasmonic photothermal therapy (PPTT) using gold nanoparticles," Lasers Med. Sci. 23, 217228 (2008).

9. W. S. Kuo, C. N. Chang, Y. T. Chang, M. H. Yang, Y. H. Chien, S. J. Chen, C. S. Yeh, "Gold nanorods in photodynamic therapy, as hyperthermia agents, and in near-infrared optical imaging," Angew. Chem., Int. Ed. 122, 2771-2775 (2010).

10. J. L. Roti Roti, "Cellular responses to hyperthermia (40-46 $\left.{ }^{\circ} \mathrm{C}\right)$ : Cell killing and molecular events," Int. J. Hyperth. 24, 3-15 (2008).

11. V. I. Bruskov, L. V. Malakhova, Z. K. Masalimov, A. V. Chernikov, "Heat-induced formation of reactive oxygen species and 8-oxoguanine, a biomarker of damage to DNA," Nucleic Acids Res. 30, 13541363 (2002).

12. H.-U. Simon, A. Haj-Yehia, F. Levi-Schaffer, "Role of reactive oxygen species (ROS) in apoptosis induction," Apoptosis 5, 415-418 (2000).

13. E. Boulais, R. Lachaine, M. Meunier, Basic mechanisms of the femtosecond laser interaction with a plasmonic nanostructure in water, Frontiers in Ultrafast Optics - Biomedical, Scientific, and Industrial Applications, A. Heisterkamp, P. R. Herman, S. Nolte, M. Meunier, Vol. 7925, pp. 79250G-5, SPIE, US (2011).

14. O. Ekici, R. K. Harrison, N. J. Durr, D. S. Eversole, M. Lee, A. Ben-Yakar, "Thermal analysis of gold nanorods heated with femtosecond laser pulses," $J$. Phys. D, Appl. Phys. 41, 185501 (2008).

15. A. Bouhelier, R. Bachelot, G. Lerondel, S. Kostcheev, P. Royer, G. P. Wiederrecht, "Surface plasmon characteristics of tunable photoluminescence in 
single gold nanorods," Phys. Rev. Lett. 95, 267405 (2005).

16. R. K. Harrison, A. Ben-Yakar, "Role of near-field enhancement in plasmonic laser nanoablation using gold nanorods on a silicon substrate," Opt. Express 18, 22556-22571 (2010).

17. J. L. Li, M. Gu, "Surface plasmonic gold nanorods for enhanced two-photon microscopic imaging and apoptosis induction of cancer cells," Biomaterials 31, 9492-9498 (2010).

18. K. Imura, H. Okamoto, "Properties of photoluminescence from single gold nanorods induced by near-field two-photon excitation," J. Phys. Chem. C 113, 11756-11759 (2009).

19. H. Wang, T. B. Huff, D. A. Zweifel, W. He, P. S. Low, A. Wei, J. X. Cheng, "In vitro and in vivo twophoton luminescence imaging of single gold nanorods," Proc. Natl. Acad. Sci. USA 102, 1575215756 (2005).

20. T. Wang, D. Halaney, D. Ho, M. D. Feldman, T. E. Milner, "Two-photon luminescence properties of gold nanorods," Biomed. Opt. Express 4, 584-595 (2013).

21. D. Floryk, J. Houštěk, "Tetramethyl rhodamine methyl ester (TMRM) is suitable for cytofluorometric measurements of mitochondrial membrane potential in cells treated with digitonin," Biosci. Rep. 19, 27-34 (1999).

22. M. G. Vander Heiden, N. S. Chandel, E. K. Williamson, P. T. Schumacker, C. B. Thompson, "Bcl$\mathrm{xL}$ regulates the membrane potential and volume homeostasis of mitochondria," Cell 91, 627-637 (1997).

23. J. J. Lemasters, T. Qian, C. A. Bradham, D. A. Brenner, W. E. Cascio, L. C. Trost, B. Herman, "Mitochondrial dysfunction in the pathogenesis of necrotic and apoptotic cell death," J. Bioenerg. Biomembr. 31, 305-319 (1999).

24. J. J. Lemasters, A. L. Nieminen, T. Qian, L. C. Trost, S. P. Elmore, Y. Nishimura, B. Herman, "The mitochondrial permeability transition in cell death: A common mechanism in necrosis, apoptosis and autophagy," BBA-Bioenerg. 1366, 177-196 (1998).

25. A. M. Alkilany, C. J. Murphy, "Toxicity and cellular uptake of gold nanoparticles: What we have learned so far?" J. Nanopart. Res. 12, 2313-2333 (2010).

26. B. D. Chithrani, A. A. Ghazani, W. C. Chan, "Determining the size and shape dependence of gold nanoparticle uptake into mammalian cells," Nano Lett. 6, 662-668 (2006).

27. L. Wang, Y. Liu, W. Li, X. Jiang, Y. Ji, X. Wu, C. Chen, "Selective targeting of gold nanorods at the mitochondria of cancer cells: Implications for cancer therapy," Nano Lett. 11, 772-780 (2010).

28. S. Link, M. A. El-Sayed, "Spectral properties and relaxation dynamics of surface plasmon electronic oscillations in gold and silver nanodots and nanorods," J. Phys. Chem. B 103, 8410-8426 (1999).

29. B. C. Stuart, M. D. Feit, S. Herman, A. M. Rubenchik, B. W. Shore, M. D. Perry, "Nanosecond-to-femtosecond laser-induced breakdown in dielectrics," Phys. Rev. B 53, 1749 (1996).

30. A. Vogel, J. Noack, G. Hüttman, G. Paltauf, "Mechanisms of femtosecond laser nanosurgery of cells and tissues," Appl. Phys. B 81, 1015-1047 (2005).

31. R. R. Letfullin, C. Joenathan, T. F. George, V. P. Zharov, "Laser-induced explosion of gold nanoparticles: Potential role for nanophotothermolysis of cancer," Future Med. 1, 473-480 (2006).

32. S. Link, C. Burda, B. Nikoobakht, M. A. El-Sayed, "Laser-induced shape changes of colloidal gold nanorods using femtosecond and nanosecond laser pulses," J. Phys. Chem. B 104, 6152-6163 (2000).

33. L. Tong, Y. Zhao, T. B. Huff, M. N. Hansen, A. Wei, J. X. Cheng, "Gold nanorods mediate tumor cell death by compromising membrane integrity," $A d v$. Mater. 19, 3136-3141 (2007). 